\title{
Two-Photon Excitation of di-4-ANEPPS for Optical Recording of Action Potentials in Rabbit Heart
}

\author{
John H. Dumas III and STEPHEN B. KNISLEY
}

(Published online: 20 April 2006)

Due to errors introduced during the typesetting process, Dr. Knisley's name was misspelled as Kinisley in the print (33:12, pp. 1802-1807) and online version (DOI: $10.1007 / \mathrm{s} 10439-005-8466-9)$ of this article. 